\title{
TUBERCULOSIS IN NEPAL: SITUATION, CHALLENGES AND WAYS FORWARD
}

\author{
Adhikari N¹, Joshi LR ${ }^{1}$,Subedi $\mathbf{B}^{1}$, Acharya $\mathrm{D}^{1}$, Adhikari $\mathbf{M}^{2}$, Thapa $\mathrm{P}^{3}$, Sultana $\mathbf{R}^{4}$, Karki KB \\ ${ }^{1}$ National Tuberculosis Program/Global Fund Program, Thimi, Bhaktapur \\ 2 District Hospital, Sindhuli \\ ${ }^{3}$ District Public Health Office, Arghakhachi \\ ${ }^{4}$ SAARC TB and HIVIAIDS Centre, Thimi, Bhaktapur
}

\section{ABSTRACT}

Introduction: Globally, tuberculosis is a major public health problem. Moreover, the emergence of drug resistant forms of TB has threatened TB prevention and treatment efforts. Despite the long history of tuberculosis prevention efforts, tuberculosis still ranks among the top ten causes of deaths in Nepal. Furthermore, Nepal being land locked with two high TB burden countries i.e. India and China, it has added difficulties to National Tuberculosis program. Hence this study aims to review the situation of National Tuberculosis Program in Nepal and explore the possible challenges and ways forward for NTP to strengthen the TB diagnostics and treatment services in Nepal.

Methods: This study is based on the review of available literatures and data sources related to tuberculosis prevention, care and control. Secondary data published by National Tuberculosis Program in the annual report for the year 2015, 2016 and 2017 were considered for the situation analysis of tuberculosis in Nepal. We used different platforms like Google scholar, PubMed to search relevant literatures.

Results: This has been a huge gap between WHO TB estimates and TB cases notification by the national system. It was 22\% in 2015, 27\% in 2016 and 29\% in 2017. However National Tuberculosis Program has maintained treatment success rate consistently above $90 \%$. Tuberculosis program has yet not achieved universal HIV testing, although HIV testing among TB patients has increased rapidly. Similarly, only 75\%, 1994 received DST out of 2601 retreatment TB cases have received DST in 2017 despite guideline suggest mandatory drug susceptibility testing for retreatment TB cases.

Conclusion: Case notification has reduced gradually. It is difficult to achieve the target envisioned by NSP 2016-21 if the current case notification trend persists. NTP needs to expand service sites ensuring minimum quality standards as well as scale up targeted intervention addressing human right issues to identify the missing TB cases. NTP needs to regulate the quality of diagnosis and treatment TB services offered by private sector.

Key words: Tuberculosis, TB, Situation, Challenges, Nepal

\section{INTRODUCTION}

Globally, tuberculosis is a major public health Problem. There were an estimated 10 million new TB cases around the world and have caused an estimated 1.3 million deaths in 2017. ${ }^{1}$ TB usually

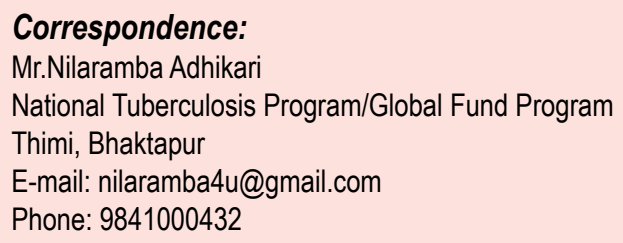

affects all the countries and all the ages; however, least developed countries bear the burden of TB the most. ${ }^{2}$ Poverty and malnutrition aggravates the burden of TB. ${ }^{3,4}$ Often, TB is cited as the poor man's disease. Globally South-East Asia region $(44 \%)$ and African region $(25 \%)$ contribute around $70 \%$ of the global TB burden. Furthermore, India $(27 \%)$, China (9\%), Indonesia (8\%), Philippines $(6 \%)$, Pakistan $(5 \%)$, Nigeria $(4 \%)$, Bangladesh $(4 \%)$ and South-Africa $(3 \%)$ alone share the twothirds of global burden. ${ }^{1,5} \mathrm{Nepal}$ is landlocked between two high TB burden countries, India and China. Moreover, Nepal shares open-border 
relationship with India. At this context, identifying, enrolling, treating TB patients and tracking them till the treatment duration and minimizing the risk of transmission to healthy population have been a difficult task to National Tuberculosis Program (NTP). Besides, the development of resistant strains by mycobacteria and comorbid conditions with other diseases like HIV and diabetes have intensified the difficulties. ${ }^{6,7}$ Hence this article aims to assess the situation of tuberculosis in Nepal, explore the challenges and opportunities for action to NTP to achieve the targets envisioned by National Strategic Plan (NSP) for Tuberculosis Prevention, Care and Control (2016-21) and End TB Strategy (2016-35). 8,9

\section{METHODS}

This study is based on the review of available literatures and data sources related to tuberculosis prevention, care and control. Both published as well as unpublished studies and reports were used. Secondary data published by National Tuberculosis Program in their annual reports for the year 2015, 2016 and 2017 were used for the situation analysis of TB program in Nepal. ${ }^{10,11,12}$ Besides, we used different search engines like Google, Google Scholar and PubMed database to retrieve articles highlighting the prevention, care and control strategies, interventions and challenges for TB program around the world. Abstract of the relevant articles were reviewed and screened for relevancy prior retrieving the full articles. Besides, references of the selected articles were also reviewed to explore additional useful articles.

\section{RESULTS AND DISCUSSION}

\section{Gap between estimated and TB case notification}

There has been a wide gap between WHO TB estimates for Nepal and TB cases notification by the national system. The gap has increased gradually; it was $22 \%$ (9,888 TB Patients, 44000 estimated vs. 34112 notified) in $2015,27 \%(11,944$ TB patients, 44000 estimated vs. 32056 notified) in 2016 and $29 \%$ (13236 TB patients, 45000 estimated vs. 31764 notified) in $2017 . .^{10,11,12}$ At this context, finding missing TB cases, especially the childhood TB cases, is major challenge for the NTP. ${ }^{13}$ These patients could have either undiagnosed or have diagnosed and not reported in the national system.
Barriers to appropriate diagnosis as well as poor linkages between the treatment and diagnosis has been identified as major obstacle which NTP needs to address. ${ }^{14}$ Besides, intervention like contact tracing of index TB patients, sputum courier at hard to reach areas, DST among retreatment TB cases need to be implemented at scale. ${ }^{15}$

\section{Decreasing diagnosis of clinically confirmed TB cases}

Furthermore, trend analysis shows diagnosis of clinically confirmed, pulmonary TB cases (PCD) has declined rapidly in comparison to bacteriologically confirmed, pulmonary TB cases (PBC) and extrapulmonary TB cases (EP). PCD cases notification had contributed $20 \%$ (6677 out of 34112 notified TB cases) of total TB cases notification in 2015 and has been decreasing thereafter (17\%; 5850 out of 32056 notified TB cases in 2016, 15\%; 5216 out of 31764 notified TB cases) in 2017). ${ }^{10,11,12}$ National data shows the gradual reduction in the number of clinically confirmed TB cases with the expansion of GeneXpert sites. However, it needs further studies to validate the association. But, studies from Nepal, Uganda and Cambodia found that clinicians werenot preferring clinical judgement for the diagnosis of TB, especially among childhood TB cases. ${ }^{16,17,18}$ Hence the focus of NTP should be placed on rational use of GeneXpert machines as well as capacity building of clinicians for clinical diagnosis of TB cases.

\section{Significant proportion of TB deaths and Lost to follow-up}

In Nepal, 9 out of 10 newly registered (new and relapse) TB patients are successfully treated. ${ }^{10,11,12} \mathrm{NTP}$ has maintained treatment success rate consistently above $90 \%$ which is above the global average. ${ }^{1}$ It was $92 \%$ in 2015 , $90 \%$ in 2016 and $91 \%$ in 2017. ${ }^{10,11,12}$ However, among retreatment TB cases, it is still below $90 \%$; the best figure was $88 \%$ in 2017 in comparison to $72 \%$ in $2015,86 \%$ in 2016. Despite, decreasing TB cases notification and high treatment success rate, TB mortality rate is consistently at around $3 \%$ which is unacceptably high given that TB deaths are prevented if it is diagnosed on time, treated with prescribed regimen till the end of treatment duration. ${ }^{12}$ Moreover, mortality due to TB is disproportionately high among male than female. ${ }^{10,11,12}$ Besides there are significant number 
of TB patients who are lost to follow-up from DOTS services. Proportion of lost to follow up TB patients in 2015 was $2 \%$ (785 out of 36050 registered TB cases). ${ }^{10}$ It has increased gradually thereafter. It was $2 \%$ (781 out of 34112 registered TB cases) in 2016, 3\% (827 out of 32056 registered TB cases) in 2017. 11,12 Their treatment status and treatment outcome are unknown. Lost to follow-up cases can serve as source for disease transmission in the community and aggravate the TB prevention efforts through the development of drug resistant forms of TB. ${ }^{19,20,21}$ Patient counselling, education and development of patient tracking mechanism to track and re-enroll such cases back into the treatment would enhance treatment adherence, reduce the risk of TB transmission. ${ }^{21}$ NTP needs to invest on the expansionas well as strengtheningits existing online NTP application like eTB, DR TB patient tracking and laboratory system.

\section{Case holding by private sectors}

The contribution of private sector in the referral and/or diagnosis of TB patients was 19\% (6552 out of 34112 notified cases) in 2015, 18\% (5969 out of 32056 notified cases) in 2016 and $23 \%$ in 2017 (7227 out of 31764 notified cases). ${ }^{10,11,12}$ It includes all TB cases referred either for diagnosis, treatment or both from private hospitals, polyclinics or pharmacies. However, true figure regarding the case holding by the private sectors in Nepal is yet unknown. Report on census of private hospitals published by CBS on 2013 shows 214 registered hospitals across the country. Majority of them were in central region (143 hospitals) followed by eastern (68 hospitals), western (59 hospitals), mid-western (21 hospitals) and far-western (10 hospitals) region respectively. ${ }^{22}$ It could have increased in the recent years. Few studies conducted in Nepal have shown patients receiving diagnostics and treatment services from the private sector irrespective of cost incurred, quality of prescribed regimen, skill of private practitioners. ${ }^{23,24}$ Moreover, majority of the private sector hospitals are out of national reporting mechanism. ${ }^{12}$ As a result, there is dearth of information regarding the TB case holding by the private sectors. Hence quality and quantity of TB diagnostic and treatment services provided by the private sector should be monitored and reported in the national system. Delivery of TB services through private sector should be guided by the policy documents like PPM strategy for TB prevention and care. ${ }^{24}$

\section{TB diagnostic services and their quality}

In Nepal, sputum microscopy, chest x-ray, GeneXpert MTB/RIF machine, culture/DST and LPA methods are the diagnostic options for TB suspects. ${ }^{12}$ NTP depends largely on the quality of these diagnostic services. Sample collection and its processing methods as well as capacity of technicians are vital to ensure the diagnostic quality. ${ }^{25}$ Based on the number of service delivery points, presumptive TB patients have comparatively greater access to sputum microscopy (604 microscopy centers) than other diagnostic services (31 GeneXpert sites, 2 culture/ DST laboratories). ${ }^{12}$ However, national trend shows false positive (+ve) and false negative (-ve) rate among the slides re-examined at regional level quality control centers is consistently high. Among the participating $(72 \%, 432$ out of 604$)$ microscopy centers (MCs) in 2017, 19 MCs (4\%) had $>5 \%$ false negative results and $10(2 \%)$ MCs had $>5 \%$ false positive results. ${ }^{10,11,12}$ Although the participation of $\mathrm{MC}$ in quality control has increased from 60\% (345 out of $576 \mathrm{MCs}$ ) in 2016 to $72 \%$ in 2017, still a significant proportion of MCs are out of quality assurance mechanism..$^{11,12}$ Similarly, error, invalid and no results account around $7 \%$ (1684 out of 23818 tests in 2016, 1480 out of 21077 tests in 2017) of the total tests in GeneXpert machine. ${ }^{11,12}$ Although the MTB (sensitive and resistant cases) detection rate has increased gradually from 19\% (4555 out of 23818 tests) in 2016 to $24 \%$ (5162 out of 21077 tests) in 2017 , yield is very low in comparison to its cost inputs and test sensitivity. ${ }^{11,12}$ Hence, greater access to and low sensitivity of microscopy have been found as major diagnostic concern. ${ }^{26}$ Expansion of diagnostic services with high sensitivity (like Culture, GeneXpert) are inevitable to reduce the number of false positive and false negative test result. But still, adequate financing, skilled human resources and addressing bio-safety issues pose additional challenges for NTP which needs to be addressed. ${ }^{27}$

\section{Poverty, malnutrition and TB HIV Diabetes Co- infection}

Tuberculosis has strong relationship with social determinants like wealth quintile, smoking and alcohol habits, housing standards, occupations. ${ }^{3,4}$ In Nepal, still one forth of the total population (25.16\%) are under the poverty line. ${ }^{28}$ Similarly, thirty-six 
percent of children under age 5 are stunted, $10 \%$ are wasted, $27 \%$ are underweight. ${ }^{29}$ Evidence has shown malnutrition as a strong predictor of TB among half of the all active TB cases. ${ }^{30,31}$ Similarly, HIV and diabetes co-infection with TB has placed an immense burden to the TB prevention and treatment efforts. It has nourished the development of active TB among the immune-compromised and has intensified TB deaths. ${ }^{32,33}$ Despite the huge efforts, HIV testing among TB patients has increased rapidly (7\% (2408 out of 34112) in 2015, $18 \%$ (5722 out of 32056) in 2016 and 54\% (17164 out of 31764) in 2017) but it is still far below the goal of universal HIV testing. 10,11,12 Hence, NTP needs to initiate as well as intensify targeted interventions to address HIV and diabetes coinfection among TB patients. Besides, NTP needs to extend coordination and coordination with different ministries, divisions and centers, government and non-government organizations, stakeholders and communities to address the social determinants to fight against TB.

\section{Burden of multi-drug resistant (MDR) TB}

Nationwide, the proportion of new cases with multidrug-resistant TB (MDR-TB) was 2.2\% among new cases and $15.4 \%$ among retreatment cases based on DRS survey carried out in 2011/12. ${ }^{12}$ However, the diagnosis of MDR TB cases in Nepal has declined gradually (379 MDR, 71 XDR TB cases in 2015, 309 MDR, 77 Pre-XDR, 17 XDR TB cases in 2016and 343 MDR, 19 XDR TB cases in 2017)..$^{10,11,12}$ While treatment success is stagnant around 70\% among MDR TB cases and 35\% among XDR TB cases. ${ }^{2}$ Studies have shown that Retreatment TB cases are comparatively more at the risk of development of drug resistant forms of TB. ${ }^{35}$ Even, TB treatment guideline developed by NTP has envisioned mandatory DST for all the retreatment TB cases. However, DST among retreatment TB cases is low. Only 75\%, 1994 received DST out of 2601 retreatment TB cases have received DST in $2017 .{ }^{12}$

\section{Adequate financing for NTP}

There were 581 microscopy centers across the country in 2015. ${ }^{10}$ It has increased gradually thereafter to 576 centers in 2016 and 604 centers in 2017..$^{11,12}$ Similarly, GeneXpert sites were also expanded gradually during this period, from 26 GeneXpert sites in 2015 to 30 sites in 2016 and 31 sites in 2017 respectively. ${ }^{10,11,12}$ But still, there is gap between TB diagnostic sites versus TB treatment sites in terms of their number. ${ }^{12}$ Expansion of service sites with quality is inevitable to achieve global and national milestones and targets on TB. Increasing coverage with quality needs adequate investment. ${ }^{36}$ However, NSP 2016-21 has already projected a funding gap of 45.8 million US dollar during its implementation. ${ }^{8}$ It would create huge challenge for Government of Nepal/NTP to achieve the targets envisioned amid the funding gap. Often quality is compromised first with funding gap. So NTP should advocate with ministry of health and finance on time and explore other funding agencies like Global Fund.

\section{Human right issues on TB}

TB is still a stigmatizing disease. Often, TB patients face stigma and discriminatory behaviour which has resulted in delay access to needed TB diagnostics and treatment services. ${ }^{37}$ Studies have identified tuberculosis related stigma as a barrier for active case finding interventions like sputum courier, contact tracing. ${ }^{37,38}$ Although NTP has initiated targeted intervention among vulnerable population like refugees, migrants, internally displaced population, prisoners, slums, malnourished children and ANC mothers, it still lacks mechanism to track and address the human right violation issues faced by TB patients. ${ }^{39} \mathrm{Hence}$ NTP as a governing body should implement interventions like empowerment activities for people with $T B$, training to health workers on right based approaches in TB diagnosis and treatment, sensitize law makers, policy makers and law enforcement agencies on TB as well as should advocate to reform any laws and regulations that hinders TB rights and services.

\section{CONCLUSION}

TB case notification has reduced gradually in Nepal. Finding missing TB cases is one of the major challenge for NTP. At this context, it is difficult for NTP to achieve the targets set by NSP 2016-21 as well as milestones and targets of End TB strategy 2016-35. Hence, NTP needs to expand service delivery sites ensuring the minimum quality standards as well as build the capacity and confidence of health workers in the diagnosis and treatment of TB patients. Besides, 
NTP should develop mechanism to regulate and monitor the quality and quantity of TB services provided by private sector. Targeted interventions addressing the human rights issues faced by TB patients support in the identification of hidden TB cases in the community. Likewise, NTP needs to explore donors and other funding agencies to reduce the funding gap.

\section{CONFLICT OF INTEREST}

\section{None}

\section{REFERENCES}

1. Global tuberculosis report 2018. Geneva: World Health Organization; 2018. Available from:http://apps.who.int/iris/bitstream/hand le/10665/274453/9789241565646-eng.pdf?ua=1

2. Dye C, Bassili A, Bierrenbach AL, BroekmansJF, et al. Measuring tuberculosis burden, trends, and the impact of control programmes. Lancet Infect Dis. 2008; 8: 233-43. Available from: https://www.ncbi. nlm.nih.gov/pubmed/18201929

3. Murray M, Oxlade O. Tuberculosis and Poverty: Why Are the Poor at Greater Risk in India? PLOS ONE 7(11): e47533. Available from: https:// journals. plos. . rg/plosone/article?id=10.1371/ journal.pone. 0047533

4. Hargreaves JR, Boccia D, Evans CA, Adato M, Petticrew $M$, et al. The social determinants of tuberculosis: from evidence to action. Am J Public Health. 2011; 101: 654-662. Available from: https:// www.ncbi.nlm.nih.gov/pmc/articles/PMC3052350/

5. The global burden of tuberculosis: results from the Global Burden of Disease Study 2015. Lancet Infect Dis. 2018; 18(3): 261-284. Available from: https:// www.ncbi.nlm.nih.gov/pmc/articles/PMC5831985/

6. Jenkins HE, Tolman AW, Yuen CM, Parr JB, et al. Incidence of multidrug-resistant tuberculosis disease in children: systematic review and global estimates. Lancet. 2014; 383(9928): 1572-9. Available from: https://www.ncbi.nlm.nih.gov/ pubmed/24671080

7. Harries $A D$, Lin $Y$, Kumar AMV, Satyanarayana $S$, et al. How can integrated care and research assist in achieving the SDG targets for diabetes, tuberculosis and HIVIAIDS? Int J Tuberc Lung Dis. 2018; 22(10): 1117-126. Available from: https:// www.ncbi.nlm.nih.gov/pubmed/30236178
8. National strategic plan for tuberculosis prevention, care and control 2016-2021. Kathmandu: National Tuberculosis Center; 2016. (36 p.) Available from: https://nepalntp.gov.np/wp-content/ uploads/2018/01/NSP-report-english-revised.pdf

9. The End TB Strategy 2016-2035. Geneva: WHO; 2015. (20 p.) Available from: http://www.who.int/tb/ End_TB_brochure.pdf?ua=1

10. Annual report 2071/72 (2015) National Tuberculosis Program Nepal. Kathmandu: National Tuberculosis Center; 2016 March. (115 p.) Available from:https:// nepalntp.gov.np/wp-content/uploads/2018/11/ TB_Annual-Report-NTPN-2015-compressedcompressed.pdf

11. Annual report 2072/73 (2016) National Tuberculosis Program Nepal. Kathmandu: National Tuberculosis Center; 2017 March. (150 p.) Available from:https:// nepalntp.gov.np/wp-content/uploads/2018/11/TB_ Annual-Report-NTPN-2016-compressed.pdf

12. Annual report $2073 / 74$ (2017) National Tuberculosis Program Nepal. Kathmandu: National Tuberculosis Center; 2018 March. (148 p.) Available from:https:// nepalntp.gov.np/wp-content/uploads/2018/03/ Final-Annual-Report-NTPN-2018.pdf

13. Improving Tuberculosis Case Detection. Geneva: Stop TB Partnership; 2015. (100 p) Available from: Available from: http://www.stoptb.org/assets/ documents/resources/publications/technical/TB_ Case_Studies.pdf

14. E Chin DP, Hanson CL. Finding the Missing Tuberculosis Patients. J Infect Dis. 2017; 216(suppl_7): S675-S678. Available from: https:// www.ncbi.nlm.nih.gov/pmc/articles/PMC5853462/

15. FGrzemska M. Finding the missing TB cases. Presented at: Child and Adolescent TB Working Group Meeting; 2017 Oct; Kigali, Rwanda. Available from: http://www.who.int/tb/areas-ofwork/children/missing_childhoodtb_cases.pdf

16. ${ }^{M}$ Creswell J, Rai B, Wali R, SudrungrotS. Introducing new tuberculosis diagnostics: the impact of Xpert MTB/RIF testing on case notifications in Nepal. INT J TUBERC LUNG DIS 19(5):545-551 Available from:https://www.ingentaconnect.com/content/ iuatld/ijtld/2015/00000019/00000005/art00010\#

17. ${ }^{G}$ Kizito S, Marquez C, Turimumahoro P, Ayakaka I, et al. Quality of care in childhood tuberculosis diagnosis at primary care clinics in Kampala, Uganda. Int J Tuberc Lung Dis. 2018; 22(10): 11961202. Available from: https://www.ncbi.nlm.nih.gov/ pubmed/30236188 
18. H Frieze JB, Yadav RP, Sokhan K, Ngak S, et al. Examining the quality of childhood tuberculosis diagnosis in Cambodia: a cross-sectional study. BMC Public Health. 2017 Mar 6;17(1):232. Available from: https://www.ncbi.nlm.nih.gov/ pubmed/28264670

19. Bam TS, Chand KB and Shrestha SD. Factors responsible for non-compliance among tuberculosis patients in Kailali District, Nepal. Journal of Nepal Health Research Council.2005; 3: 51-57. Available from:http://jnhrc.com.np/index.php/jnhrc/article/ view/111

20. Wares DF, Singh S, Acharya AK and Dngi R. Non-adherence to tuberculosis treatment in the eastern Tarai of Nepal.International Journal of Tuberculosis and Lung Disease. 2003; 7(4): 327335. Available from:https://www.ncbi.nlm.nih.gov/ pubmed/12729337

21. Bam TS, Gunneberg C, Chamroonsawasdi K, Bam DS, Aalberg $O$, et al. Factors affecting patient adherence to DOTS in urban Kathmandu, Nepal. Int J Tuberc Lung Dis 2006; 10: 270-276. Available from: https://www.ncbi.nlm.nih.gov/ pubmed/165627061

22. A Report on Census of Private Hospitals in Nepal 2013. Kathmandu: Central Bureau of Statistics; 2014. (74 p.) Available from:http://cbs.gov. np/image/data/2015/A\%20Report\%20on\%20 Census $\% 20$ of $\% 20$ Private $\% 20$ Hospitals $\% 20$ in $\% 20$ Nepal\%202013.pdf

23. 'Nepal AK, Shrestha A, Baral SC, Bhattarai R,AryalY.Tuberculosis practices among private medical practitioners in Kaski district, Nepal. Int J Infect Microbial 2012;1(2);68-75. Available from: https://www.nepjol.info/index.php/IJIM/article/ view/7085

24. JNewell JN, Pande SB, Baral SC, Bam DS, Malla P. Control of tuberculosis in an urban setting in Nepal: public-private partnership. Bull World Health Organ. 2004; 82(2): 92-8. Available from: https:// www.ncbi.nlm.nih.gov/pubmed/15042230

25. Guide for providing technical support to TB laboratories in low- and middle-income countries. Geneva: Stop TB Partnership; 2017. $(96$ p.) Available from: https://www.kncvtbc. org/uploaded/2017/08/Guide_for_Providing_ Technical_Support.pdf

26. KNema V. Tuberculosis diagnostics: Challenges and opportunities. Lung India. 2012; 29(3): 259266. Available from: https://www.ncbi.nlm.nih.gov/ pmc/articles/PMC3424866/?report=printable
27. Joshi B, Lestari T, Graham SM, Baral SC, et al. The implementation of Xpert MTB/RIF assay for diagnosis of tuberculosis in Nepal: A mixedmethods analysis. PLoS One. 2018; 13(8): e0201731. Available from: https://www.ncbi.nlm. nih.gov/pubmed/30096174

28. A Poverty in Nepal (Brief Report based on Nepal living Standard Survey -Third 2066/67). Kathmandu: Central Bureau of Statistics; 2012. (24 p.) Available from: http://cbs.gov.np/image/data/ Surveys/poverty $\% 20 \mathrm{in} \% 20 \mathrm{Nepal} \% 202010-11 /$ CBS\%20view\%20on\%20poverty\%20in\%20Nepal. pdf

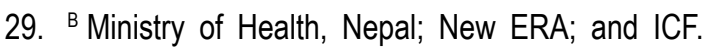
Nepal Demographic and Health Survey 2016. Kathmandu, Nepal: Ministry of Health, Nepal; 2017. Available from: https://www.dhsprogram. com/pubs/pdf/fr336/fr336.pdf

30. c. Pai M, Daftary A, Satyanarayana S. TB control: challenges and opportunities for India. Trans R Soc Trop Med Hyg. 2016; 110: 158-160.Available from: https://www.ncbi.nlm.nih.gov/pubmed/26884494

31. Bhargava A, Sharma A, Oxlade $O$ et al. Undernutrition and the incidence of tuberculosis in India: national and subnational estimates of the population-attributable fraction related to undernutrition. Natl Med J India 2014; 27: 12833. Available from: https://www.ncbi.nlm.nih.gov/ pubmed/25668081

32. Sah SK, Verma SC, Bhattarai R, Bhandari K, Bhatta GK. Surveillance of HIV Infection among Patients with Tuberculosis in Nepal. SAARC Journal of Tuberculosis, Lung Diseases and HIVIAIDS. 2015; 12(1): 25-30. Available from:https://www.nepjol. info/index.php/SAARCTB/article/view/15939

33. Maharjan B, ChaliseHN, Thapa M. Tuberculosis and Diabetes Mellitus Comorbidity among the Ageing Population: A Threat to the Public Health System of Nepal. J Nepal Health Res Counc 2018 AprJun;16(39):110-7. Available from:http://www.jnhrc. com.np/index.php/jnhrc/article/view/1092/692

34. Surveillance on Anti Tuberculosis Drug Resistance among TB Patients in Nepal 2011/12. Kathmandu: Nepal Anti Tuberculosis Association (NATA)/ German Nepal Tuberculosis Project (GENETUP); $2012(25 p$.

35. Marahatta SB. Multi-drug resistant tuberculosis burden and risk factors: An update. Kathmandu University Medical Journal. 2010; 8(1): 116-125. Available from:https://www.nepjol.info/index.php/ KUMJ/article/viewFile/6238/5086 
36. Kruk ME, Gage AD, Arsenault C, Jordan K, et al. High-quality health systems in the Sustainable Development Goals era: time for a revolution. The Lancet Global Health. 2018; 6(11): Pe1196-e1252. Available from: https://www.thelancet.com/ journals/langlo/article/PIIS2214-109X(18)30386-3/ fulltext

37. DingakeOBK. Human Rights, TB, Legislation, and Jurisprudence. Health Hum Rights. 2017; 19(1): 305-309. Available from: https://www.ncbi.nlm.nih. gov/pmc/articles/PMC5473060/
38. Hough MA, Ggita J, Turimumahoro P, Meyer AJ, et al. 'Something so hard': a mixed-methods study of home sputum collection for tuberculosis contact investigation in Uganda. Int $\mathrm{J}$ Tuberc Lung Dis. 2018; 22(10): 1152-1159. Available from: https:// www.ncbi.nlm.nih.gov/pubmed/30236182

39. Sub Recipient Program Implementation Guideline (Second Edition). Kathmandu: National Tuberculosis Center; 2018. (49 p.) Available from: https://nepalntp.gov.np/wp-content/ uploads/2018/06/TB_SR_Implementation_ Guideline_Final_8-6-2018.pd 